\title{
SOLAR SYSTEM AS A RADIO TELESCOPE BY THE FORMATION OF VIRTUAL LENSES ABOVE AND BELOW
}

\author{
Vishnu Vardhan. $\mathrm{Ch}^{1}$ \\ Department of E.C.E, AL-AMAN College of Engineering, Visakhapatnam, India, vbobby4@gmail.com
}

\begin{abstract}
The boundaries of multiverse are almost infinite and so the thirst of mankind for latest technology .man has startled many principles of space and nature by gaining them facing all fatal difficulties. Time is one quantity which is considered to be fast happening, but when it comes to happenings in space it is faster. All what we look into space is past; under least possible cases we could see the present. So, there has a necessicity for us to keep a big eye on making our search for extra-galactic recourses or extra-celestial life forms etc with equal speeds of space time. for this to be achieved by us we need to make some drastic changes in our telescope usage and we have to adopt technological up gradation and we should no longer make our telescope concentrate on the same celestial body for days continuously, indeed mili seconds should be enough to grab the required information .
\end{abstract}

Index Terms- RTS, LEO, MEO, GEO, RT

\section{INTRODUCTION}

\section{LOW EARTH ORBIT (LEO):}

Typical Uses: Satellite phone, Military, Observation

Orbiting the earth at roughly $160-500$ miles altitude, low earth orbit (LEO) satellites complete one orbit roughly every 90 minutes [1]. This means that they are fast moving $(>17,000 \mathrm{mph})$ and sophisticated ground equipment must be used to track the satellite. This makes for expensive antennas that must track the satellite and lock to the signal while moving.

\section{MIDDLE EARTH ORBIT (MEO)}

Typical Uses: Weather Satellites, Observation

Most of the satellites in this orbital altitude circle the earth at approximately 6,000 to 12,000 miles above the earth in an elliptical orbit around the poles of the earth. As the earth rotates, these satellites cover the entire surface of the earth. Fewer satellites are required to create coverage for the entire earth, as these satellites are higher and have a larger footprint.[2]

\section{GEOSTATIONARY/GEOSYNCHRONOUS (GEO) :}

Typical Uses: Television, Long Distance Communications,

Internet

At 22,240 miles above the earth, craft inserted into orbit over the equator and traveling at approximately 6,880 miles per hour around the equator following the earth's rotation. This allows these satellites to maintain their relative position over the earth's surface[3]. Since the satellite follows the earth, and takes 24 hours to complete it's orbit around the earth, geostationary orbits are also called geosynchronous.

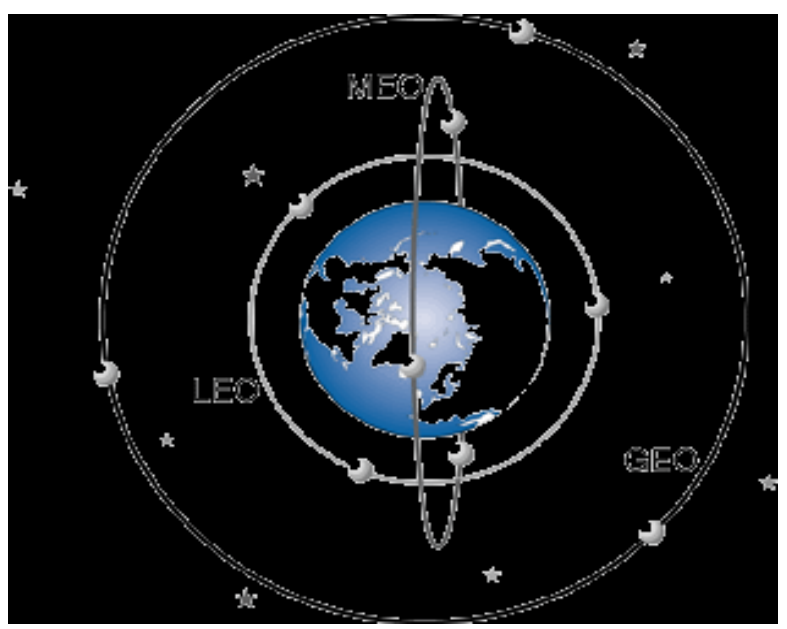

Figure 1: This explains about the altitudes of GEO, MEO, LEO

TABLE 1: ORBITAL DISTANCES

\begin{tabular}{|l|c|c|}
\hline \multicolumn{1}{|c|}{ Orbit Distance } & Miles & Km \\
\hline Low Earth Orbit (LEO) & $100-500$ & $160-$ \\
1,400
\end{tabular}




\section{KEPLERS LAWS OF PLANEARY MOTION}

\section{Law 1:-}

Each planet orbits the sun in an elliptical path with the

sun at one focus
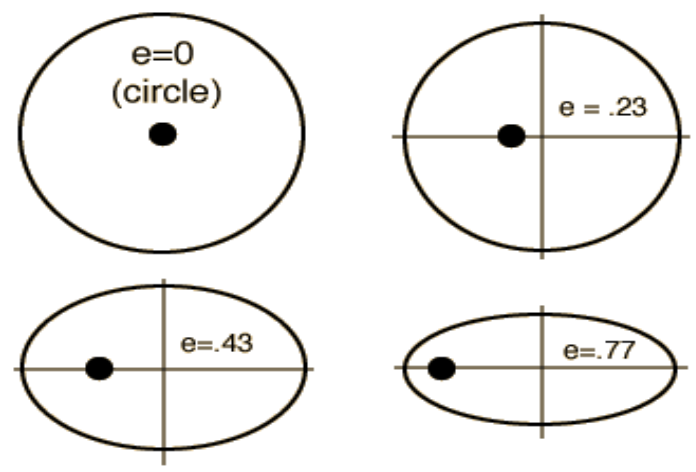

Figure 2: elliptical paths with varying eccentrics

\section{Law 2:-}

The radius vector ( from sun to planet ) sweeps out equal areas in equal time intervals

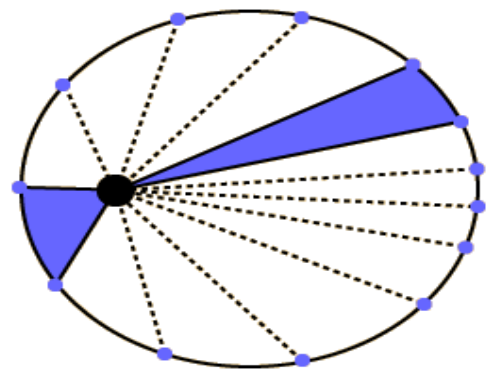

Figure 3: The positions of the planet in its orbit

Law 3:-

The square of the period is proportional to the cube of the semi-major axis of the orbit . (i.e. )

$$
\mathrm{T}^{2}=\mathrm{k}^{3}
$$

For some constant $\mathrm{k}$

\section{POSITIONING OF RADIO TELESCOPIC}

\section{SATELLITES AT DIFFERENT POINTS IN THE ORBIT FOLLOWED BY THE EARTH AROUND}

\section{THE SUN}

To have a better understanding about the topic mentioned above lets first review with the Keplers laws. Keplers 1st law sates that "all planets move in elliptical orbits with a star at any one of the foci ", Keplers 2nd law sates that "the line that connects a planet to the star sweeps out equal areas in equal times "[4].

The model proposed in this paper describes about positioning of radio telescopic satellites in the orbit followed by different planets around the sun[5]. The very purpose behind this model is to rectify the fact that RT satellites cannot view the universe in the way they should, this is because of earth's atmosphere which is acting as a filter, hindering most of the data reception by the RT satellites back down on earth. Viewing through radio telescopes is an elegant way of studying multiverse[6]. But Still the cloud cover and other ecological cycles on the earth interferes the working efficiency of radio telescopes. The ozone layer does its job of shielding us from UV, gamma, $x$ ray and other harmful radiations, which are the basic requisites for a radio telescope so as to have an effective way of analyzing a celestial object.

To eliminate this defect, this paper suggests of launching radio telescopic satellites into the orbits followed by the planets around the sun. These would revolve with a velocity similar to that of the planet but equally spaced and located at different positions on the entire orbit. It takes some amount of duration to reach perihelion if started at aphelion and vice-versa. During this entire period the radio telescopes on planet fails to grasp other radiations incident on to the rest of the orbit. This drawback can be overcome by positioning RT satellites at as many different positions as possible in the orbits of planets aiding to view the multiverse specks where RTs on the planet cannot (because of course of revolution) [7].

The planets like earth, mars, Jupiter, Saturn, Uranus, Neptune etc whose orbits can be used to launch radio telescopic satellites to make a virtual lense above and below the solar system (refer picture-4). the number of satellites of needed to be launched in the planets orbit is independent to that particular orbit for example the planet earth needs 12 satellites in its orbit for every one month one radio telescopic satellite should be launched so that the orbit is completely surveyed similarly it depends on the length of the orbit and the velocity with which the RTS revolve in the orbit [8] .

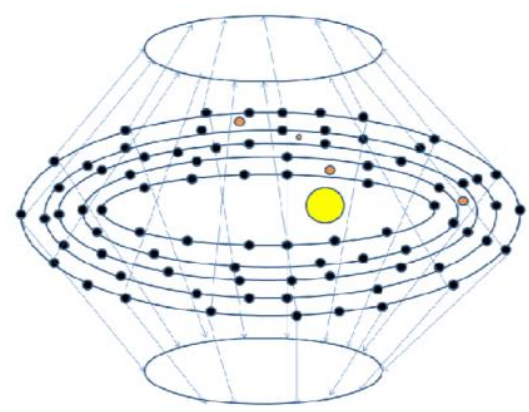

Figure4 -: view of orbits around the star in which the RTS are positioned and thus a virtual telescope is formed. 
In the above picture the black circles are radio telescopic satellites, the red circles are also called radio telescopic satellites but their functionality is a bit different and indeed they are used for a different purpose and are not utilized in the formation of the lense[9]. These red color circles represent radio telescopic signal transmitter satellites which are used to bring back the data collected from different planets to the earth. And, finally the yellow colored circle is the star[10]. The thus formed lense is very much powerful and it could be used to collect the data from distant celestial bodies.

The main reason behind using radio telescopes is, radio waves has less frequency and so they can travel for longer distances, so obviously the data collected will be about the celestial bodies present beyond the limits of optical telescopes capacity[11] .

Table 2: Different Statistics Of Rts Revolving Round The Sun in the orbit of earth.

\begin{tabular}{|c|c|c|c|c|}
\hline $\begin{array}{l}\mathrm{N} \\
\mathrm{O}\end{array}$ & $\begin{array}{l}\text { SATE } \\
\text { L- } \\
\text { LITE } \\
\text { NAM } \\
\text { E }\end{array}$ & $\begin{array}{l}\text { SEMI- } \\
\text { MAJOR } \\
\text { AXIS ( in } \\
\mathrm{km} \text { ) }\end{array}$ & $\begin{array}{l}\text { INCLI } \\
\text { NATI } \\
\text { ON } \\
\text { (degre } \\
\text { es) }\end{array}$ & $\begin{array}{l}\text { ECCEN } \\
\text {-TRICITY }\end{array}$ \\
\hline 1 & RTS-1 & $\begin{array}{l}14959826 \\
2\end{array}$ & 7.155 & $\begin{array}{l}0.01671021 \\
9\end{array}$ \\
\hline 2 & RTS-2 & $\begin{array}{l}14959826 \\
2\end{array}$ & 7.155 & $\begin{array}{l}0.01671021 \\
9\end{array}$ \\
\hline 3 & RTS-3 & $\begin{array}{l}14959826 \\
2\end{array}$ & 7.155 & $\begin{array}{l}0.01671021 \\
9\end{array}$ \\
\hline 4 & RTS-4 & $\begin{array}{l}14959826 \\
2\end{array}$ & 7.155 & $\begin{array}{l}0.01671021 \\
9\end{array}$ \\
\hline 5 & RTS-5 & $\begin{array}{l}14959826 \\
2\end{array}$ & 7.155 & $\begin{array}{l}0.01671021 \\
9\end{array}$ \\
\hline 6 & RTS-6 & $\begin{array}{l}14959826 \\
2\end{array}$ & 7.155 & $\begin{array}{l}0.01671021 \\
9\end{array}$ \\
\hline 7 & RTS-7 & $\begin{array}{l}14959826 \\
2\end{array}$ & 7.155 & $\begin{array}{l}0.01671021 \\
9\end{array}$ \\
\hline 8 & RTS-8 & $\begin{array}{l}14959826 \\
2\end{array}$ & 7.155 & $\begin{array}{l}0.01671021 \\
9\end{array}$ \\
\hline 9 & RTS-9 & $\begin{array}{l}14959826 \\
2\end{array}$ & 7.155 & $\begin{array}{l}0.01671021 \\
9\end{array}$ \\
\hline $\begin{array}{l}1 \\
0\end{array}$ & $\begin{array}{l}\text { RTS- } \\
10\end{array}$ & $\begin{array}{l}14959826 \\
2\end{array}$ & 7.155 & $\begin{array}{l}0.01671021 \\
9\end{array}$ \\
\hline $\begin{array}{l}1 \\
1\end{array}$ & $\begin{array}{l}\text { RTS- } \\
11\end{array}$ & $\begin{array}{l}14959826 \\
2\end{array}$ & 7.155 & $\begin{array}{l}0.01671021 \\
9\end{array}$ \\
\hline $\begin{array}{l}1 \\
2\end{array}$ & $\begin{array}{l}\text { RTS- } \\
12\end{array}$ & $\begin{array}{l}14959826 \\
2\end{array}$ & 7.155 & $\begin{array}{l}0.01671021 \\
9\end{array}$ \\
\hline
\end{tabular}

Table 3: Different Statistics Of Rts Revolving Round The Sun in the orbit of Jupiter

\begin{tabular}{|c|c|c|c|c|}
\hline $\begin{array}{l}\mathrm{S} \\
\mathrm{L} \\
\vdots \\
\mathrm{N} \\
\mathrm{O}\end{array}$ & $\begin{array}{l}\text { SATE } \\
\text { L- } \\
\text { LITE } \\
\text { NAM } \\
\text { E } \\
\end{array}$ & $\begin{array}{l}\text { SEMI- } \\
\text { MAJOR } \\
\text { AXIS ( in } \\
\mathrm{km} \text { ) }\end{array}$ & $\begin{array}{l}\text { INCL } \\
\text { INAT } \\
\text { ION } \\
\text { (degre } \\
\text { es) }\end{array}$ & $\begin{array}{l}\text { ECCEN } \\
\text {-TRICITY }\end{array}$ \\
\hline 1 & RTS-1 & $\begin{array}{l}7.7857 * 1 \\
0^{\wedge} 8\end{array}$ & 1.305 & 0.0484 \\
\hline 2 & RTS-2 & $\begin{array}{l}7.7857 * 1 \\
0^{\wedge} 8\end{array}$ & 1.305 & 0.0484 \\
\hline 3 & RTS-3 & $\begin{array}{l}7.7857 * 1 \\
0^{\wedge} 8\end{array}$ & 1.305 & 0.0484 \\
\hline 4 & RTS-4 & $\begin{array}{l}7.7857 * 1 \\
0^{\wedge} 8\end{array}$ & 1.305 & 0.0484 \\
\hline 5 & RTS-5 & $\begin{array}{l}7.7857 * 1 \\
0^{\wedge} 8\end{array}$ & 1.305 & 0.0484 \\
\hline 6 & RTS-6 & $\begin{array}{l}7.7857 * 1 \\
0^{\wedge} 8\end{array}$ & 1.305 & 0.0484 \\
\hline 7 & RTS-7 & $\begin{array}{l}7.7857 * 1 \\
0^{\wedge} 8\end{array}$ & 1.305 & 0.0484 \\
\hline 8 & RTS-8 & $\begin{array}{l}7.7857 * 1 \\
0^{\wedge} 8\end{array}$ & 1.305 & 0.0484 \\
\hline 9 & RTS-9 & $\begin{array}{l}7.7857 * 1 \\
0^{\wedge} 8 \\
\end{array}$ & 1.305 & 0.0484 \\
\hline $\begin{array}{l}1 \\
0\end{array}$ & $\begin{array}{l}\text { RTS- } \\
10\end{array}$ & $\begin{array}{l}7.7857 * 1 \\
0^{\wedge} 8\end{array}$ & 1.305 & 0.0484 \\
\hline $\begin{array}{l}1 \\
1\end{array}$ & $\begin{array}{l}\text { RTS- } \\
11\end{array}$ & $\begin{array}{l}7.7857 * 1 \\
0^{\wedge} 8\end{array}$ & 1.305 & 0.0484 \\
\hline $\begin{array}{l}1 \\
2\end{array}$ & $\begin{array}{l}\text { RTS- } \\
12\end{array}$ & $\begin{array}{l}7.7857 * 1 \\
0^{\wedge} 8\end{array}$ & 1.305 & 0.0484 \\
\hline $\begin{array}{l}1 \\
3\end{array}$ & $\begin{array}{l}\text { RTS- } \\
13\end{array}$ & $\begin{array}{l}7.7857 * 1 \\
0^{\wedge} 8\end{array}$ & 1.305 & 0.0484 \\
\hline $\begin{array}{l}1 \\
4\end{array}$ & $\begin{array}{l}\text { RTS- } \\
14\end{array}$ & $\begin{array}{l}7.7857 * 1 \\
0^{\wedge} 8\end{array}$ & 1.305 & 0.0484 \\
\hline
\end{tabular}

Table 4: Different Statistics Of Rts Revolving Round The Sun in the orbit of Saturn.

\begin{tabular}{|l|l|l|l|l|}
\hline S & SATE & SEMI- & INCLI & ECCEN \\
L & L- & MAJOR & NATIO & - \\
$:$ & LITE & AXIS ( in & N \\
N & NAM & $\mathrm{km})$ & TRICITY \\
O & E & & s) & \\
\hline 1 & RTS-1 & $\begin{array}{l}1.43353^{*} 1 \\
0^{\wedge} 9\end{array}$ & 2.484 & 0.0542 \\
. & & $\begin{array}{l}1.43353^{*} 1 \\
0^{\wedge 9}\end{array}$ & 2.484 & 0.0542 \\
\hline 2 & RTS-2 & & \\
\hline. & & $1.43353^{*} 1$ & 2.484 & 0.0542 \\
\hline 3 & RTS-3 & \multicolumn{2}{|l}{} \\
\hline
\end{tabular}




\begin{tabular}{|c|c|c|c|c|}
\hline & & $0^{\wedge} 9$ & & \\
\hline 4 & RTS-4 & $\begin{array}{l}1.43353 * 1 \\
0^{\wedge} 9\end{array}$ & 2.484 & 0.0542 \\
\hline 5 & RTS-5 & $\begin{array}{l}1.43353 * 1 \\
0^{\wedge} 9\end{array}$ & 2.484 & 0.0542 \\
\hline 6 & RTS-6 & $\begin{array}{l}1.43353 * 1 \\
0^{\wedge} 9\end{array}$ & 2.484 & 0.0542 \\
\hline 7 & RTS-7 & $\begin{array}{l}1.43353 * 1 \\
0^{\wedge} 9\end{array}$ & 2.484 & 0.0542 \\
\hline 8 & RTS-8 & $\begin{array}{l}1.43353 * 1 \\
0^{\wedge} 9\end{array}$ & 2.484 & 0.0542 \\
\hline 9 & RTS-9 & $\begin{array}{l}1.43353 * 1 \\
0^{\wedge} 9\end{array}$ & 2.484 & 0.0542 \\
\hline $\begin{array}{l}1 \\
0\end{array}$ & $\begin{array}{l}\text { RTS- } \\
10\end{array}$ & $\begin{array}{l}1.43353 * 1 \\
0^{\wedge} 9\end{array}$ & 2.484 & 0.0542 \\
\hline $\begin{array}{l}1 \\
1\end{array}$ & $\begin{array}{l}\text { RTS- } \\
11\end{array}$ & $\begin{array}{l}1.43353^{*} 1 \\
0^{\wedge} 9\end{array}$ & 2.484 & 0.0542 \\
\hline $\begin{array}{l}1 \\
2\end{array}$ & $\begin{array}{l}\text { RTS- } \\
12\end{array}$ & $\begin{array}{l}1.43353 * 1 \\
0^{\wedge} 9\end{array}$ & 2.484 & 0.0542 \\
\hline $\begin{array}{l} \\
3\end{array}$ & $\begin{array}{l}\text { RTS- } \\
13\end{array}$ & $\begin{array}{l}1.43353 * 1 \\
0^{\wedge} 9\end{array}$ & 2.484 & 0.0542 \\
\hline $\begin{array}{l}1 \\
4\end{array}$ & $\begin{array}{l}\text { RTS- } \\
14\end{array}$ & $\begin{array}{l}1.43353 * 1 \\
0^{\wedge} 9\end{array}$ & 2.484 & 0.0542 \\
\hline $\begin{array}{l}1 \\
5\end{array}$ & $\begin{array}{l}\text { RTS- } \\
15\end{array}$ & $\begin{array}{l}1.43353 * 1 \\
0^{\wedge} 9\end{array}$ & 2.484 & 0.0542 \\
\hline $\begin{array}{l}1 \\
6\end{array}$ & $\begin{array}{l}\text { RTS- } \\
16\end{array}$ & $\begin{array}{l}1.43353^{*} 1 \\
0^{\wedge} 9\end{array}$ & 2.484 & 0.0542 \\
\hline
\end{tabular}

Table 5: Different Statistics Of Rts Revolving Round The Sun in the orbit of Uranus

\begin{tabular}{|c|c|c|c|c|}
\hline $\begin{array}{l}\mathrm{S} \\
\mathrm{L} \\
\vdots \\
\mathrm{N} \\
\mathrm{O}\end{array}$ & $\begin{array}{l}\text { SATE } \\
\text { L- } \\
\text { LITE } \\
\text { NAM } \\
\text { E }\end{array}$ & $\begin{array}{l}\text { SEMI- } \\
\text { MAJOR } \\
\text { AXIS ( in } \\
\mathrm{km} \text { ) }\end{array}$ & $\begin{array}{l}\text { INCL } \\
\text { INAT } \\
\text { ION } \\
\text { (degre } \\
\text { es) }\end{array}$ & $\begin{array}{l}\text { ECCEN } \\
\text {-TRICITY }\end{array}$ \\
\hline 1 & RTS-1 & $\begin{array}{l}2.87426^{*} \\
10^{\wedge} 9\end{array}$ & 0.769 & 0.0086 \\
\hline 2 & RTS-2 & $\begin{array}{l}2.87426^{*} \\
10^{\wedge} 9\end{array}$ & 0.769 & 0.0086 \\
\hline 3 & RTS-3 & $\begin{array}{l}2.87426^{*} \\
10^{\wedge} 9\end{array}$ & 0.769 & 0.0086 \\
\hline 4 & RTS-4 & $\begin{array}{l}2.87426^{*} \\
10^{\wedge} 9\end{array}$ & 0.769 & 0.0086 \\
\hline 5 & RTS-5 & $\begin{array}{l}2.87426^{*} \\
10^{\wedge} 9\end{array}$ & 0.769 & 0.0086 \\
\hline 6 & RTS-6 & $\begin{array}{l}2.87426^{*} \\
10^{\wedge} 9\end{array}$ & 0.769 & 0.0086 \\
\hline
\end{tabular}

\begin{tabular}{|c|c|c|c|c|}
\hline 7 & RTS-7 & $\begin{array}{l}2.87426^{*} \\
10^{\wedge} 9\end{array}$ & 0.769 & 0.0086 \\
\hline 8 & RTS-8 & $\begin{array}{l}2.87426^{*} \\
10^{\wedge} 9\end{array}$ & 0.769 & 0.0086 \\
\hline 9 & RTS-9 & $\begin{array}{l}2.87426^{*} \\
10^{\wedge} 9\end{array}$ & 0.769 & 0.0086 \\
\hline $\begin{array}{l}1 \\
0\end{array}$ & $\begin{array}{l}\text { RTS- } \\
10\end{array}$ & $\begin{array}{l}2.87426^{*} \\
10^{\wedge} 9\end{array}$ & 0.769 & 0.0086 \\
\hline $\begin{array}{l}1 \\
1\end{array}$ & $\begin{array}{l}\text { RTS- } \\
11\end{array}$ & $\begin{array}{l}2.87426^{*} \\
10^{\wedge} 9\end{array}$ & 0.769 & 0.0086 \\
\hline $\begin{array}{l}1 \\
2\end{array}$ & $\begin{array}{l}\text { RTS- } \\
12\end{array}$ & $\begin{array}{l}2.87426^{*} \\
10^{\wedge} 9\end{array}$ & 0.769 & 0.0086 \\
\hline $\begin{array}{l}1 \\
3\end{array}$ & $\begin{array}{l}\text { RTS- } \\
13\end{array}$ & $\begin{array}{l}2.87426^{*} \\
10^{\wedge} 9\end{array}$ & 0.769 & 0.0086 \\
\hline $\begin{array}{l}1 \\
4\end{array}$ & $\begin{array}{l}\text { RTS- } \\
14\end{array}$ & $\begin{array}{l}2.87426^{*} \\
10^{\wedge} 9\end{array}$ & 0.769 & 0.0086 \\
\hline $\begin{array}{l}1 \\
5\end{array}$ & $\begin{array}{l}\text { RTS- } \\
15\end{array}$ & $\begin{array}{l}2.87426^{*} \\
10^{\wedge} 9\end{array}$ & 0.769 & 0.0086 \\
\hline $\begin{array}{l}1 \\
6\end{array}$ & $\begin{array}{l}\text { RTS- } \\
16\end{array}$ & $\begin{array}{l}2.87426^{*} \\
10^{\wedge} 9\end{array}$ & 0.769 & 0.0086 \\
\hline $\begin{array}{l}1 \\
7\end{array}$ & $\begin{array}{l}\text { RTS- } \\
17\end{array}$ & $\begin{array}{l}2.87426^{*} \\
10^{\wedge} 9\end{array}$ & 0.769 & 0.0086 \\
\hline $\begin{array}{l}1 \\
8\end{array}$ & $\begin{array}{l}\text { RTS- } \\
18\end{array}$ & $\begin{array}{l}2.87426^{*} \\
10^{\wedge} 9\end{array}$ & 0.769 & 0.0086 \\
\hline
\end{tabular}

Table 6: Different Statistics Of Rts Revolving Round The Sun in the orbit of Neptune

\begin{tabular}{|c|c|c|c|c|}
\hline $\begin{array}{l}\mathrm{S} \\
\mathrm{L}\end{array}$ & $\begin{array}{l}\text { SAT } \\
\text { EL- } \\
\text { LITE } \\
\text { NAM } \\
\text { E }\end{array}$ & $\begin{array}{l}\text { SEMI- } \\
\text { MAJOR } \\
\text { AXIS ( } \\
\text { in km ) }\end{array}$ & $\begin{array}{l}\text { INCLINA } \\
\text { TION } \\
\text { (degrees) }\end{array}$ & $\begin{array}{l}\text { ECCEN } \\
- \\
\text { TRICIT } \\
\mathrm{Y}\end{array}$ \\
\hline 1 & $\begin{array}{l}\text { RTS- } \\
1\end{array}$ & $\begin{array}{l}4.49506 \\
* 10^{\wedge} 9\end{array}$ & 1.769 & 0.0086 \\
\hline 2 & $\begin{array}{l}\text { RTS- } \\
2\end{array}$ & $\begin{array}{l}4.49506 \\
* 10^{\wedge} 9\end{array}$ & 1.769 & 0.0086 \\
\hline 3 & $\begin{array}{l}\text { RTS- } \\
3\end{array}$ & $\begin{array}{l}4.49506 \\
* 10^{\wedge} 9\end{array}$ & 1.769 & 0.0086 \\
\hline 4 & $\begin{array}{l}\text { RTS- } \\
4\end{array}$ & $\begin{array}{l}4.49506 \\
* 10^{\wedge} 9\end{array}$ & 1.769 & 0.0086 \\
\hline 5 & $\begin{array}{l}\text { RTS- } \\
5\end{array}$ & $\begin{array}{l}4.49506 \\
* 10^{\wedge} 9\end{array}$ & 1.769 & 0.0086 \\
\hline 6 & $\begin{array}{l}\text { RTS- } \\
6\end{array}$ & $\begin{array}{l}4.49506 \\
* 10^{\wedge} 9\end{array}$ & 1.769 & 0.0086 \\
\hline 7 & RTS- & 4.49506 & 1.769 & 0.0086 \\
\hline
\end{tabular}




\begin{tabular}{|c|c|c|c|c|}
\hline & 7 & $* 10^{\wedge} 9$ & & \\
\hline 8 & $\begin{array}{l}\text { RTS- } \\
8\end{array}$ & $\begin{array}{l}4.49506 \\
* 10^{\wedge} 9\end{array}$ & 1.769 & 0.0086 \\
\hline 9 & $\begin{array}{l}\text { RTS- } \\
9\end{array}$ & $\begin{array}{l}4.49506 \\
* 10^{\wedge} 9\end{array}$ & 1.769 & 0.0086 \\
\hline $\begin{array}{l}1 \\
0\end{array}$ & $\begin{array}{l}\text { RTS- } \\
10\end{array}$ & $\begin{array}{l}4.49506 \\
* 10^{\wedge} 9\end{array}$ & 1.769 & 0.0086 \\
\hline $\begin{array}{l}1 \\
1\end{array}$ & $\begin{array}{l}\text { RTS- } \\
11\end{array}$ & $\begin{array}{l}4.49506 \\
* 10^{\wedge} 9\end{array}$ & 1.769 & 0.0086 \\
\hline $\begin{array}{l}1 \\
2\end{array}$ & $\begin{array}{l}\text { RTS- } \\
12\end{array}$ & $\begin{array}{l}4.49506 \\
* 10^{\wedge} 9\end{array}$ & 1.769 & 0.0086 \\
\hline $\begin{array}{l}1 \\
3\end{array}$ & $\begin{array}{l}\text { RTS- } \\
13\end{array}$ & $\begin{array}{l}4.49506 \\
* 10^{\wedge} 9\end{array}$ & 1.769 & 0.0086 \\
\hline $\begin{array}{l}1 \\
4\end{array}$ & $\begin{array}{l}\text { RTS- } \\
14\end{array}$ & $\begin{array}{l}4.49506 \\
* 10^{\wedge} 9\end{array}$ & 1.769 & 0.0086 \\
\hline $\begin{array}{l}1 \\
5\end{array}$ & $\begin{array}{l}\text { RTS- } \\
15\end{array}$ & $\begin{array}{l}4.49506 \\
* 10^{\wedge} 9\end{array}$ & 1.769 & 0.0086 \\
\hline $\begin{array}{l}1 \\
6\end{array}$ & $\begin{array}{l}\text { RTS- } \\
16\end{array}$ & $\begin{array}{l}4.49506 \\
* 10^{\wedge} 9\end{array}$ & 1.769 & 0.0086 \\
\hline $\begin{array}{l}1 \\
7\end{array}$ & $\begin{array}{l}\text { RTS- } \\
17\end{array}$ & $\begin{array}{l}4.49506 \\
* 10^{\wedge} 9\end{array}$ & 1.769 & 0.0086 \\
\hline $\begin{array}{l}1 \\
8 \\
\end{array}$ & $\begin{array}{l}\text { RTS- } \\
18\end{array}$ & $\begin{array}{l}4.49506 \\
* 10^{\wedge} 9\end{array}$ & 1.769 & 0.0086 \\
\hline $\begin{array}{l}1 \\
9 \\
\end{array}$ & $\begin{array}{l}\text { RTS- } \\
19 \\
\end{array}$ & $\begin{array}{l}4.49506 \\
* 10^{\wedge} 9 \\
\end{array}$ & 1.769 & 0.0086 \\
\hline $\begin{array}{l}2 \\
0\end{array}$ & $\begin{array}{l}\text { RTS- } \\
20\end{array}$ & $\begin{array}{l}4.49506 \\
* 10^{\wedge} 9\end{array}$ & 1.769 & 0.0086 \\
\hline
\end{tabular}

\section{SIMILATION RESULTS}

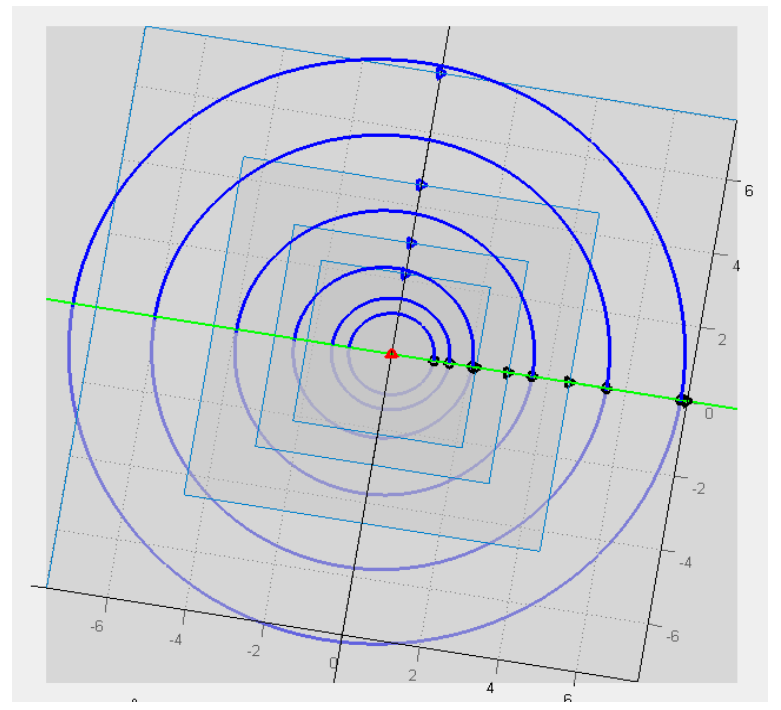

Picture-5: top view of orbits of planets into which RTS will be launched to form a virtual telescope

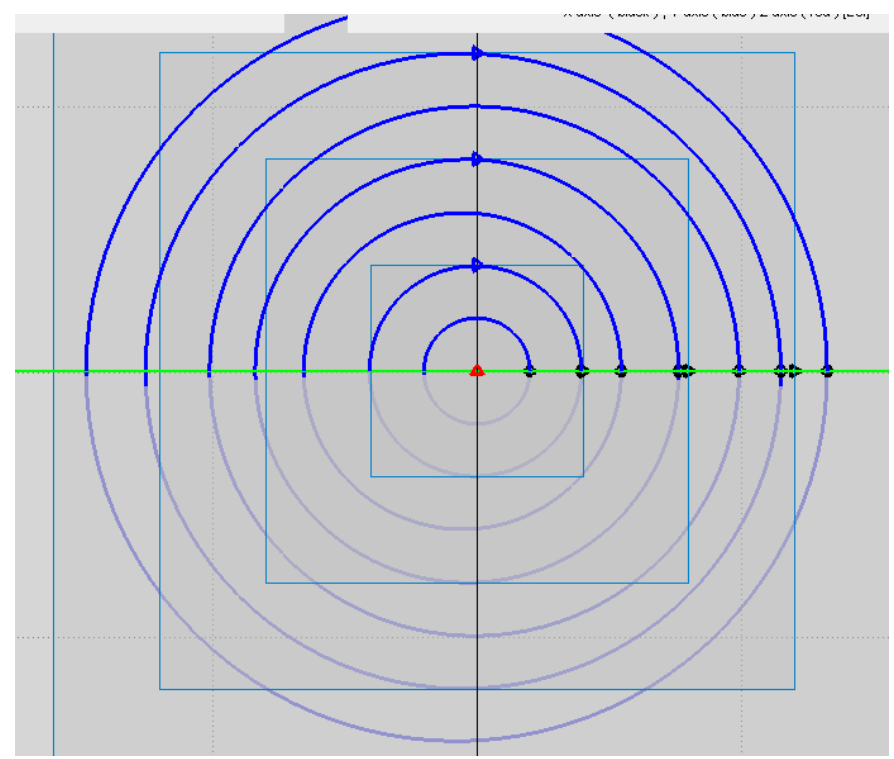

Picture-6: top view of orbit of solar system ( here in this picture, seven orbits are graphed) 


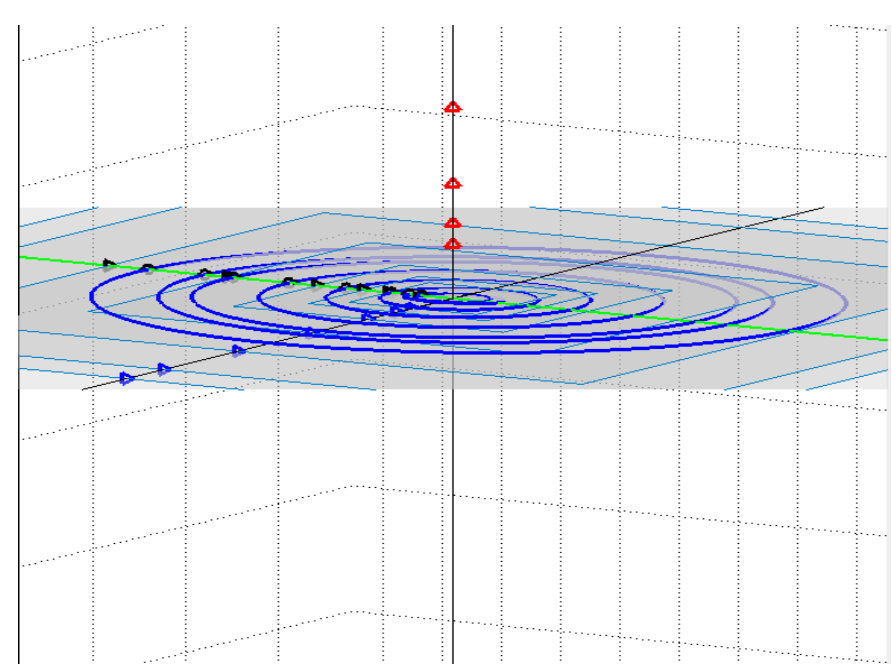

Picture-7: side view of the orbits of the planets into which RTS will be launched

\section{ACKNOWLEDGMENTS}

The author likes to thank his parents and sister for giving financial aid in publishing this paper

\section{REFERENCES}

[1] "Significant Achievements in Space Communications and Navigation, 1958-1964". NASA-SP-93. NASA. 1966. pp. 3032. Retrieved 2009-10-31.

[2] F. Ananasso, F. Delli Pricoli, "The Role of Satellites in Personal Communications Services," IEEE Journal on Selected Areas in Communications," V 13, N 2, (February 1995), pp.180-195.

[3] M.H. Hadjitheodosiou, F.P. Coakley and B.G. Evans, "Multiacess Protocols for a Multiservice VSAT Network. Center for Satellite Engineering Research, University of Surrey, Guildford, UK.

[4] A. Guntsch. Analysis of the ATDMA/PRMA++ Protocol in a Mobile Satellite Environment.In Proceedings 46th IEEE Vehicular Technology Conference '96, pp. 1225-1229, Atlanta, U.S.A., April 1996.

[5] Bruce R. Elbert, "Ths Satellite Communication applications handbook",1997 Artech House, Inc, MA.

[6] Elbert, Bruce R, The satellite communication applications handbook, Boston, MA: Artech House, 1997.

[7] Feldman, Phillip M., An overview and comparison of demand assignment multiple access (DAMA) concepts for satellite communications networks Santa Monica, CA: RAND, 1996. Student Project Report, 2000.

[8] Wertz, James R. and Wiley J.Larson, Space Mission Analysis and Design, Microcosm Press, El Segundo CA 1999, pg 533-586.

[9] Daniel E. Friedman, Masters Thesis: "Error Control for Satellite and Hybrid Communication Networks", directed by Daniel E. Friedman, 1995
[10] John S. Baras, ATM in Hybrid Networks, Center for Satellite and Hybrid Communication Networks, 1996

[11] Vivek Arora, Narin Suphasindhu, John S. Baras, Douglas Dillon, "Effective Extensions of Internet in Hybrid SatelliteTerrestrial Networks", University of Maryland at College Park \& Hughes Network Systems, Inc., 1996

\section{BIOGRAPHY:}

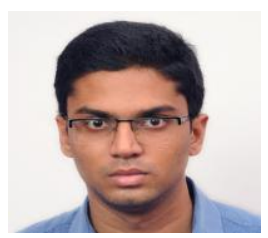

Chakravarm Vishnu Vardhan has completed under graduation in the year 2012. He has published four research papers in international journals, Published a book titled "Mankind Predator on Nature " whose ISBN is 978-93-8216361-9 presented a paper in International Conference, presented papers in eleven national level symposiums, attended robotic work shops . 